\title{
Humanness beliefs about behavior: An index and comparative human-nonhuman behavior judgments
}

\author{
Samuel G. Wilson • Nick Haslam
}

Published online: 20 September 2012

(C) Psychonomic Society, Inc. 2012

\begin{abstract}
Social psychological research suggests that two distinct dimensions describe lay conceptions of humanness: a species-typical sense (i.e., human nature) and a speciesunique sense (i.e., human uniqueness). Although these two senses of humanness have been discerned among psychological traits and states, there has been no systematic research into lay beliefs about the humanness of human behaviors. Using a range of 60 prosocial, nonsocial, and antisocial behaviors, it was demonstrated that people discriminate between species-typical and species-unique behaviors and that the capacity to perform species-unique behaviors distinguishes humans from animals, whereas the capacity to perform species-typical behaviors distinguishes humans from robots. Behaviors that exemplify the two senses of humanness are identified, and data representing rankings, raw scores, and $z$-scores in two indices of species typicality and species uniqueness are provided. Taken together, these findings expand our understanding of lay conceptions of humanness and provide researchers of humanness with a wider range of validated stimuli to probe the boundaries of humanity.
\end{abstract}

Keywords Humanness $\cdot$ Human nature $\cdot$ Dehumanization . Nonhumans $\cdot$ Behavior

Electronic supplementary material The online version of this article (doi:10.3758/s13428-012-0252-7) contains supplementary material, which is available to authorized users.

S. G. Wilson · N. Haslam

Melbourne School of Psychological Sciences,

University of Melbourne,

Parkville, VIC, Australia

Present Address:

S. G. Wilson $(\bowtie)$

School of Psychology and Psychiatry, Monash University,

Clayton, Victoria 3800, Australia

e-mail: samuel.wilson@monash.edu
Conceptions of humanness that emphasize the human discontinuity with the nonhuman order go back thousands of years (Plumwood, 2002). These perceived discontinuities between humans and nonhumans persevere in laypeople's beliefs about humanness and are associated with judgments about who is fully human and who is less than fully human (Castano \& Giner-Sorolla, 2006; Goff, Eberhardt, Williams, \& Jackson, 2008; Haslam, Bain, Douge, Lee, \& Bastian, 2005; Leyens et al., 2000).

Recent years have witnessed the proliferation of social psychological research into laypeople's conceptions of humanness. This research typically focuses on specific psychological traits and states, such as emotions (Demoulin et al., 2004), personality traits (Haslam et al., 2005; Haslam, Bastian, \& Bissett, 2004), and mental capacities (H. M. Gray, Gray, \& Wegner, 2007). To date, there has been no systematic research into lay beliefs about the humanness of behaviors or the behavioral similarities and distinctions between humans, animals, and machines.

The purpose of this study is to expand our understanding of humanness to the behavioral realm. In particular, the purpose of this study is to examine beliefs about the humanness of diverse human behaviors and how people discriminate between humans and nonhumans behaviorally. These findings will be useful to dehumanization and anthropomorphism researchers, as well as to social cognition researchers more generally. Beyond psychology, these findings will be useful to bioethics scholars concerned with whether dignity and moral worth are attributable to how an agent behaves (an extrinsic performance criterion) or what an agent is (an intrinsic worth criterion) (Brooks, 2002; Somerville, 2006; see Wilson \& Haslam, 2009, for a review).

\section{Conceptions of humanness}

The longest debated frontier of human identity in Western culture has been between humans and animals (Fernández- 
Armesto, 2004), and it is this frontier that has historically informed Western conceptions of what it means to be human (J. Gray, 2003). The boundary between humans and nonhuman animals is not, however, the only boundary to inform our understanding of what it is to be human. Another salient boundary is that between humans and machines (Hampshire, 1991; Turkle, 1991).

Social psychological research attests to the psychological reality of these very basic ways of understanding what it means to be human (Demoulin et al., 2004; H. M. Gray et al., 2007; Haslam et al., 2005; Loughnan \& Haslam, 2007; Loughnan, Haslam, \& Kashima, 2009). These two humannonhuman oppositions inform not only our understanding of what it means to be fully human or animal- or machine-like, but also our beliefs about the proper treatment of the humanized and dehumanized. For example, humanness judgments, measured via attributions of psychological traits and states, influence judgments of moral agency and patiency (Bastian, Laham, Wilson, Haslam, \& Koval, 2011), as well as our willingness to help (Vaes, Paladino, Castelli, Levens, \& Giovanazzi, 2003) and harm (Bandura, 2002; Bandura, Barbaranelli, Caprara, \& Pastorelli, 1996; Bandura, Underwood, \& Fromson, 1975) others.

Social psychological research into laypeople's conceptions of humanness has focused on three related categories of human attributes: emotions, personality traits, and mental capacities. What is striking about this body of research is that, despite reflecting distinct theoretical perspectives, broadly comparable results have emerged about lay conceptions of humanness and how our specific senses of humanness separate humans from animals and machines, respectively.

\section{Humanness beliefs about emotions}

Demoulin et al. (2004) demonstrated that laypeople distinguish between emotions that are shared with animals (nonuniquely human emotions) and emotions that are considered uniquely human (uniquely human emotions), which parallel the distinctions made by emotion scientists (e.g., Ekman, 1992; Epstein, 1984). Nonuniquely human emotions (e.g., anger, surprise) are judged as innate, visible to outside observers, caused by external events, and of short duration (Demoulin et al., 2004; Rodriguez et al., 2005). By contrast, uniquely human emotions (e.g., love, guilt) are judged as invisible to observers, generated internally via thinking, experienced over a long duration, morally informative, and acquired through socialization (Demoulin et al., 2004; Rodriguez et al., 2005). The focus on emotions that are shared with animals (i.e., nonuniquely human emotions) and uniquely human emotions indicates that humanness is conceptualized in a way that emphasizes the human-animal boundary.
Humanness beliefs about personality traits

Haslam et al.'s $(2005,2004)$ research into conceptions of humanness, as manifest in personality traits, suggests that there are two distinct senses of humanness: a species-unique sense, termed human uniqueness (HU) and a species-typical sense, termed human nature $(\mathrm{HN})$. Human uniqueness, by definition, refers to those traits that distinguish humans from animals, exemplified by refinement, moral sensibility, selfcontrol, and rationality (Haslam et al., 2005). Uniquely human traits are judged as acquired through learning, as requiring maturity for their expression, not prevalent in the population, and culturally specific (Haslam et al., 2005). In contrast, HN reflects the biologically-based human essence - the fundamental or essential attributes of the human species - some of which may be shared with animals. Traits judged as part of $\mathrm{HN}$ reflect emotional responsiveness, prosocial warmth, cognitive openness, and individuality (Haslam et al., 2005). HN traits tend to be regarded as innate, prevalent within cultures, universal across cultures, and positive or socially desirable (Haslam et al., 2005, 2004). Dehumanization research provides insights into how the two senses of humanness distinguish humans from nonhumans. For example, Loughnan and Haslam (2007) demonstrated that HN traits are associated, explicitly and implicitly, with animals more than with machines and that uniquely human traits are associated with machines more than with animals. Moreover, the denial to people of traits that reflect HN leads them to be likened to machines, whereas the denial to people of traits that reflect HU leads them to be likened to animals (Loughnan et al., 2009).

\section{Humanness beliefs about mental capacities}

Research by H. M. Gray et al. (2007) into laypeople's beliefs about mental capacities suggests that there are two dimensions of mind perception, termed agency and experience. Agency is exemplified by the capacity for language, reason, self-control, and morality. This dimension parallels the findings obtained for uniquely human emotions and traits. By contrast, the experience dimension involves capacities for consciousness, primary emotions, and basic appetites. This dimension parallels the judgments obtained for personality traits that reflect HN. Consistent with emotion- and trait-based research, agentic capacities highlight the human-animal boundary and experiential capacities highlight the human-machine boundary: adult humans are seen as differing from animals on the agency dimension and from robots on the experience dimension (H. M. Gray et al., 2007).

To summarize, distinct lines of research converge on the idea that there are two basic ways of thinking about humanness, which emphasize species-typical and species-unique qualities, respectively. $\mathrm{HN}$ is a species-typical sense of 
humanness that emphasizes our continuity with nature. HN is understood as the human essence - innate, immutable, and universal (Haslam et al., 2005). HN is characterized by capacities for primary consciousness (i.e., being aware of things in the world), basic or primary emotions (e.g., pleasure, fear), and appetites (e.g., hunger), as well as capacities for forming and maintaining affectionate relationships (interpersonal warmth), learning from experience (cognitive openness), and individuality and self-determination (H. M. Gray et al., 2007; Haslam et al., 2005, 2004). HN marks the boundary between humans and inert nonhumans - robots and machines. Consistent with this, denying $\mathrm{HN}$ to people characterizes them as machine-like (Loughnan \& Haslam, 2007; Loughnan et al., 2009).

$\mathrm{HU}$ is a species-unique sense of humanness that emphasizes our transcendence of nature. HU is not understood as a natural human inheritance but, rather, as acquired through social learning and enculturation (Haslam et al., 2005). HU is characterized by capacities for higher-order consciousness (i.e., being conscious of being conscious, semantic ability) and uniquely human emotions (e.g., love, shame), as well as cognitively complex capacities for rationality (thought, planning), language, and the self-control and moral sensibility required for civility (Demoulin et al., 2004; H. M. Gray et al., 2007; Haslam et al., 2005, 2004). HU, by definition, marks the boundary between humans and animals. Consistent with this, denying HU to people characterizes them as animal-like (Loughnan \& Haslam, 2007; Loughnan et al., 2009).

Although previous attribute-based research has demonstrated that lay conceptions of humanness distinguish between a species-typical sense (i.e., $\mathrm{HN}$ ) and a speciesunique sense (i.e., HU), research to date has not examined whether these two senses of humanness are discerned when the focal attributes are behaviors. Examining humanness beliefs about behaviors is crucial given that basic behavioral representations of people undergird more complex traitbased representations. The present study addresses this gap in our understanding of lay concepts of humanness. Demonstrating that $\mathrm{HN}$ and $\mathrm{HU}$ are discerned among behaviors, that these dimensions are distinct, and that the conceptual characterizations of these dimensions parallel those found in previous attribute-based research would strengthen the argument that two distinct senses of humanness underlie conceptions of humanness in Western folk psychology.

Our first hypothesis predicted that the two senses of humanness should be discerned among behaviors. Consistent with Haslam et al. (2005, 2004), HN and HU judgments about behavior should be distinct; evidenced by no association or a weak negative correlation between the dimensions. Our second hypothesis predicted that conceptual judgments associated with $\mathrm{HN}$ and $\mathrm{HU}$ in the behavioral domain will parallel the foregoing characterizations of $\mathrm{HN}$ and HU. That is, HN behaviors should be judged to involve the experience of emotion, as positive, prevalent within a population, universal across cultures, and innate (i.e., not learned). By contrast, HU behaviors should be judged as cognitively demanding, morally informative, emergent late in development, acquired through learning, and culturally specific (i.e., not universal). Our third hypothesis predicted that humans should be distinguished from animals and machines along the $\mathrm{HU}$ and $\mathrm{HN}$ dimensions, respectively. Specifically, the more a behavior reflects HU, the less animals should perform it, relative to humans. Similarly, the more a behavior reflects HN, the less robots should perform it, relative to humans. Given that humans and animals enact behaviors grounded in our shared mammalian evolution (e.g., sleeping) and that machines enact behaviors that mimic uniquely human intellective behaviors (e.g., performing calculations), it is unclear whether humans will be differentiated from animals and machines on $\mathrm{HN}$ and $\mathrm{HU}$, respectively. Accordingly, hypotheses are not advanced about these relationships.

\section{Method}

\section{Participants}

Participants were 103 undergraduates (69 women and 34 men) ranging in age from 17 to $40(M=20.4)$. They were recruited for a study of beliefs about human behavior and participated in order to fulfill a course research participation requirement.

\section{Materials}

All participants completed a questionnaire in which they rated a list of behaviors on a set of ten items. Sixty behaviors were used, which reflected a wide variety of basic and complex prosocial, asocial, and antisocial behaviors. The criterion guiding the selection of behaviors was that they could be performed by humans and either animals or robots. The behaviors were sampled from a variety of books on animal behavior (e.g., MacLean, 1990) and robotics (e.g., Brooks, 2002). The behaviors selected were described in an abstract, general manner (e.g., "greeting gestures" rather than "waving back when a friend waves") so as to be amenable to judgments about their performance by animals and robots.

Two items were adapted from Haslam et al. (2005) to assess the two senses of humanness (HN: "This behavior is an aspect of human nature"; HU: "This behavior is performed exclusively by human beings; that is, it is not performed by other species"). Four items were based on past work by Haslam et al. $(2005,2004)$ on predictors of $\mathrm{HN}$ 
(emotion: "The performance of this behavior involves the experience of emotions"; positivity: "How desirable or positive is this behavior?"; prevalence: "A large proportion of the population performs this behavior"; universality: "This behavior is performed universally; that is, it is performed in all cultures"). Four items were adapted from Demoulin et al. (2004) to assess predictors of HU (age of emergence: "It is possible to perform this behavior at a young age" [reverse scored]; cognition: "This behavior is primarily cognitive; that is, it requires a lot of thinking"; morality: "The fact that someone performs this behavior gives us an idea about their moral nature, about their morality"; social learning: "This behavior is learned; that is, people are not born knowing how to perform this behavior"). All ten items were rated on 7-point Likert scales ranging from 1 (strongly disagree) to 7 (strongly agree).

Finally, two items assessed beliefs about whether two categories of nonhumans could perform the behaviors (animals: "Please indicate the degree to which animals perform the following behaviors compared to humans"; robots: "Please indicate the degree to which robots (i.e., robots, automata, and machines) perform the following behaviors compared to humans"). These two items were rated on 5point Likert scales ranging from 1 (much less than humans) to 5 (much more than humans), with the option of answering " 0 " when the behavior was judged as not applicable to animals or robots.

Two versions of the questionnaire were created. The first version presented the HN question first and the HU question last, and the second version reversed this order of presentation. Within each questionnaire version, the eight other conceptual judgment items were randomized. The two items that assessed judgments about the performance of behaviors by animals and automata relative to humans were placed at the end of the questionnaire booklet.

\section{Procedure}

Groups of 2-12 participants completed the questionnaire in a laboratory setting under the supervision of the researcher, taking less than $45 \mathrm{~min}$.

\section{Results}

\section{Reliability}

The analyses of ten conceptual judgments in this study were conducted at the level of the behavior. To determine whether participants were able to make these judgments reliably, the correlation between each participant's ratings and the average ratings by all participants on the dimension was calculated, following the practice of Rothbart and Park (1986).
Participants whose judgments correlated either negatively or near zero (less than .10) with the mean score on each dimension of humanness were excluded. Thirty judgments that correlated either negatively or near zero were identified and eliminated, with no more than ten from any given dimension. The number of participants contributing to the score of each dimension varied from 93 to 103 , which means that fewer than $10 \%$ of the judges were excluded per dimension (see Table 1).

Reliability was assessed by Cronbach's alpha and the intraclass correlation coefficient. Although this procedure usually looks at the degree of interrelatedness among $i$ items, over $j$ judges, in this instance items and judges are reversed such that what is under consideration is the degree of agreement among judges over the items (i.e., the 60 behaviors). A two-way absolute agreement, average measures random model was selected, and the reliability coefficients ranged from .97 to .99 for both the original sample ( $N=103$ per dimension) and the sample screened for unreliable judges $(N=93-103$ per dimension), indicating high levels of agreement (see Table 1). The data from the latter sample were used in the analyses presented here.

\section{Correlations between conceptual judgments}

Using the reliable judges, the mean value for the 60 behaviors on each judgment dimension was computed for use in the correlation analysis. Mean ratings of the 60 behaviors on the study items were computed by aggregating across the number of reliable judges within each of the ten conceptual judgments. The correlations among the conceptual judgments are presented in Table 2. HN and HU were weakly negatively associated $(r=-.37, p<.01)$, indicating that humanness beliefs, although somewhat related, are distinct.

\section{Beliefs about behavior}

HN was positively associated with positivity $(r=.82, p<.01)$, prevalence $(r=.90, p<.1)$ and universality $(r=.66, p<.01)$, but not emotionality ( $r=.10$, n.s.). The negative association found between $\mathrm{HN}$ and social learning $(r=-.43, p<.01)$ is consistent with the hypothesis that $\mathrm{HN}$ would be judged as innate. In sum, four of the five proposed conceptual correlates of HN were supported. HU was positively associated with cognition $(r=.65, p<.01)$, but, contrary to expectations, not morality ( $r=-.04$, n.s.). Consistent with expectations, HU was positively associated with age of emergence $(r=.34, p<.01)$ and social learning $(r=.81, p<.01)$ and negatively associated with universality $(r=-.51, p<.01)$. In sum, four of the five proposed conceptual correlates of UH were supported. These findings indicate that $\mathrm{HN}$ and $\mathrm{HU}$ are characterized by distinct conceptual profiles. 
Table 1 Number of judges and reliability values before and after deletion of unreliable judges

\begin{tabular}{|c|c|c|c|c|c|c|}
\hline Belief dimension & Original $N$ & $\begin{array}{l}\text { Original alpha } \\
\text { value }\end{array}$ & $\begin{array}{l}\text { Original } \\
\text { ICC value }\end{array}$ & Final $N$ & $\begin{array}{l}\text { Final alpha } \\
\text { value }\end{array}$ & $\begin{array}{l}\text { Final ICC } \\
\text { value }\end{array}$ \\
\hline Human nature & 103 & .97 & .97 & 93 & .98 & .97 \\
\hline Human uniqueness & 103 & .99 & .99 & 101 & .99 & .99 \\
\hline Emotion & 103 & .99 & .99 & 102 & .99 & .99 \\
\hline Positive & 103 & .99 & .99 & 103 & .99 & .99 \\
\hline Prevalence & 103 & .99 & .99 & 101 & .99 & .99 \\
\hline Universality & 103 & .97 & .96 & 93 & .97 & .96 \\
\hline Age of emergence & 103 & .99 & .98 & 102 & .99 & .98 \\
\hline Cognition & 103 & .99 & .99 & 103 & .99 & .99 \\
\hline Morality & 103 & .99 & .99 & 101 & .99 & .99 \\
\hline Social learning & 103 & .98 & .98 & 102 & .98 & .98 \\
\hline Animal behavior & 103 & .99 & .99 & 103 & .99 & .99 \\
\hline Automata behavior & 103 & .99 & .99 & 103 & .99 & .99 \\
\hline
\end{tabular}

ICC, intraclass correlation coefficient

\section{Behaviors that exemplify $\mathrm{HN}$ and $\mathrm{HU}$}

To emphasize the status of a given behavior's $\mathrm{HN}$ and $\mathrm{HU}$ score within the distribution of 60 behaviors, the nonstandardized ratings of reliable participants were converted to $z$ scores. (Rankings, raw scores, and $z$-scores of participants' $\mathrm{HN}$ and $\mathrm{HU}$ ratings of the 60 behaviors and $z$-scores for these behaviors on the eight conceptual judgments are available in Tables 7-9 in a supplemental file.) The top 10 behaviors that were awarded high $\mathrm{HN}$ ratings (i.e., more than one standard deviation above the mean) were, in rank order, sleeping, sex, establishing and maintaining affectionate relationships, eating/drinking, forming social groups, verbal communication, locomotion, making facial expressions, maternal care of young, and speaking/talking. The comparable list for $\mathrm{HU}$ was reading, studying, writing, numerical reasoning, growing food, performing calculations, speaking/talking, verbal reasoning, making art, and self-harm.

To further examine whether behaviors cluster together on the basis of their humanness ratings, a cluster analysis was run on the 60 behaviors using their $\mathrm{HN}$ and HU ratings. A hierarchical cluster analysis using Ward's method produced six clusters, which were significantly different in the main (see Table 3). Cluster 1 was defined by behaviors that reflect capacities for language and reason. Cluster 2 was defined by basic survival (e.g., eating, sleeping) and prosocial behaviors directed at maintaining close, communal relationships (e.g., helping family members). Cluster 3 was defined by

Table 2 Correlations among human nature (HN) and human uniqueness (HU) beliefs and eight conceptual judgments

\begin{tabular}{|c|c|c|c|c|c|c|c|c|c|}
\hline $\begin{array}{l}\text { Conceptual } \\
\text { judgment }\end{array}$ & $1(\mathrm{HN})$ & 2 (HU) & 3 (Pos) & 4 (Emo) & 5 (Uni) & 6 (Prev) & 7 (Mat) & $8(\mathrm{Cog})$ & 9 (Mor) \\
\hline
\end{tabular}

\begin{tabular}{|c|c|c|c|c|c|c|c|c|c|}
\hline \\
\hline \multicolumn{10}{|l|}{$\begin{array}{l}\text { 1. HN } \\
\text { 2. HU }\end{array}$} \\
\hline 3. Positive (Pos) & $.82 * *$ & -.01 & & & & & & & \\
\hline 4. Emotional (Emo) & .10 & -.04 & $-.27 *$ & & & & & & \\
\hline 5. Universal (Uni) & $.66^{* *}$ & $-.51 * *$ & $.36^{* *}$ & -.12 & & & & & \\
\hline 6. Prevalence (Prev) & $.90 * *$ & -.24 & $.78 * *$ & -.20 & $.57 * *$ & & & & \\
\hline 7. Maturity (Mat) & $-.54 * *$ & $.34 * *$ & $-.33 * *$ & .15 & $-.53 *$ & $-.59 * *$ & & & \\
\hline 8. Cognition (Cog) & -.12 & $.65^{* *}$ & .19 & .13 & $-.35^{* *}$ & -.10 & $.43 * *$ & & \\
\hline 9. Morality (Mor) & $-.44 * *$ & -.04 & $-.60 * *$ & $.75^{* *}$ & $-.31 *$ & $-.51 * *$ & $.37 * *$ & -.08 & \\
\hline 10. Social learning & $-.43 * *$ & $.81^{* *}$ & -.06 & -.14 & $-.50 * *$ & $-.33 *$ & $.51 * *$ & $.76 * *$ & .03 \\
\hline
\end{tabular}

$* p<.05$

$* * p<.01$ 
Table 3 Cluster analysis of 60 behaviors as defined by human nature and human uniqueness ratings

Cluster 1: Language and Reason

Cluster 2: Survival and Prosocial I (communal relationships with family and friends)

Reading

Eating/drinking

Writing

Speaking/talking

Sleeping

Sex

Numerical reasoning

Verbal reasoning

Performing calculations

Playing thinking games

Studying

Making art

Growing food

Cluster 3:

Prosocial II (hierarchical and transactional relationships with others)

Planning/organizing

Following rules and making rules

Negotiating

Working

Performing complicated tasks

Helping strangers

Judging others

Calculated aggression

Practising

Singing

Making music

Cluster 5:

Applied reason and Prosocial III (superficial coordination with others)

Greeting gestures

Verbal communication

Acting/pretending

Paternal care of young

Teaching

Solving problems

Making things (e.g., objects)

Negative behaviors are italicized

deliberative behaviors involved in social coordination in hierarchical and transactional relationships (e.g., negotiation, following rules). Cluster 4 was defined by behaviors involved in the manipulation of the natural environment (e.g., manipulating objects, building shelter) and social dominance (e.g., fighting, impulsive aggression). Cluster 5 was defined by applied reason and superficial prosocial behaviors (e.g., greeting gestures, solving problems). Cluster 6 was defined by antisocial behaviors involving harm to self and others (e.g., self-harm, bullying).

There were significant differences between clusters in terms of HN, $F(5,54)=22.97, p<.001$; HU, $F(5,54)=$ $116.87, p<.001$; positivity, Welch's $F(5,22.21)=13.88, p<$ .001 ; prevalence, Welch's $F(5,23.14)=10.94, p<.001$; universality, $F(5,54)=6.19, p<.001$; age of emergence, $F$ $(5,54)=2.71, p<.05$; thinking, $F(5,54)=11.67, p<.001$; social learning, Welch's $F(5,23.77)=23.35, p<.001$; and morality, Welch's $F(5,23.17)=7.27, p<.001$. There were no difference between the clusters in terms of the extent to which they involved the experience of emotions, Welch's $F(5$, $24.09)=1.77$, n.s. (see Table 4 for descriptive statistics).

If $\mathrm{HN}$ behaviors are emotional, positive, prevalent, universal, and innate and HU behaviors require thought and are morally informative, socially learned, emergent late in development, and culturally specific, these patterns of judgments should be apparent when clusters that reflect $\mathrm{HN}$ and HU, respectively, are compared. This task is better achieved by comparing clusters with specific humanness profiles than by a series of post hoc tests. The six clusters of behaviors that emerged in the cluster analysis can be classified into three distinct categories: high HN, high HU (cluster 1); high HN, low HU (clusters 2-5); and low HN, low HU (cluster 6). No clusters had a low HN, high HU profile.

\section{High-HU versus low-HU clusters}

A series of $t$-tests demonstrated that the high-HU cluster (cluster 1) had higher HU ratings than the low-HU clusters (clusters 2-6), but comparable $\mathrm{HN}$ ratings. A comparison between the clusters to examine the conceptual correlates of HU could therefore be made. As compared with the low-HU cluster, behaviors in the high-HU cluster received higher cognition and social learning ratings and lower universality ratings. Contrary to expectations, there were no differences between the clusters in age of emergence. Further, the lowHU cluster was judged as more morally informative than the high HU-cluster, rather than less. In sum, three out of five expectations about the characteristics of HU behaviors were met (see Table 5).

\section{High-HN versus low-HN clusters}

A series of $t$-tests demonstrated that the high-HN clusters (clusters 1-5) had higher $\mathrm{HN}$ ratings than the low-HN cluster (cluster 6) but comparable HU ratings. A comparison between the clusters to examine the conceptual correlates of HN could therefore be made. As compared with the low-HN 
Table 4 Means and standard deviations of humanness and related conceptual judgments across six clusters of behavior

\begin{tabular}{|c|c|c|c|c|c|c|c|c|c|c|}
\hline \multirow[t]{2}{*}{ Cluster } & \multicolumn{2}{|c|}{ Sense of humanness } & \multicolumn{8}{|c|}{ Conceptual judgments } \\
\hline & $\mathrm{HN}$ & $\mathrm{HU}$ & Emotional & Positive & Prevalent & Universal & Learned & Cognitive & Maturity & Morality \\
\hline 1 & $4.96(0.49)$ & $5.71(0.45)$ & $3.99(1.20)$ & $5.81(0.45)$ & $5.23(0.82)$ & $5.53(0.65)$ & $5.76(0.46)$ & $5.74(0.81)$ & $3.77(0.69)$ & $2.97(0.47)$ \\
\hline 2 & $5.92(0.24)$ & $1.65(0.43)$ & $4.89(1.68)$ & $6.31(0.33)$ & $6.37(0.51)$ & $6.48(0.36)$ & $3.00(0.77)$ & $3.45(1.17)$ & $2.66(1.37)$ & $3.57(1.50)$ \\
\hline 3 & $4.97(0.39)$ & $3.88(0.39)$ & $4.80(1.00)$ & $5.11(1.31)$ & $5.21(0.80)$ & $5.95(0.35)$ & $5.03(0.36)$ & $5.27(0.85)$ & $3.85(0.76)$ & $4.32(1.25)$ \\
\hline 4 & $4.81(0.59)$ & $1.70(0.31)$ & $4.11(1.62)$ & $4.21(1.60)$ & $4.94(1.41)$ & $6.05(0.39)$ & $3.96(0.70)$ & $3.46(0.92)$ & $3.23(1.20)$ & $3.61(1.61)$ \\
\hline 5 & $5.40(0.33)$ & $2.84(0.30)$ & $4.75(1.12)$ & $5.88(0.67)$ & $5.63(0.69)$ & $5.98(0.74)$ & $4.50(0.47)$ & $5.07(1.16)$ & $3.41(0.96)$ & $3.93(0.84)$ \\
\hline 6 & $3.76(0.66)$ & $3.47(.075)$ & $5.31(0.84)$ & $2.39(1.58)$ & $3.44(1.24)$ & $5.46(0.46)$ & $4.74(0.16)$ & $4.03(0.53)$ & $4.10(0.53)$ & $5.33(1.19)$ \\
\hline
\end{tabular}

The number of behaviors per cluster is as follows: cluster 1 (10 behaviors); cluster 2 (12 behaviors); cluster 3 (11 behaviors); cluster 4 (11 behaviors); cluster 5 ( 7 behaviors); cluster 6 ( 9 behaviors). HN, human nature; HU, human uniqueness

cluster, the high-HN cluster received higher positivity, prevalence, and universality ratings, but not lower social learning ratings. Contrary to expectations, the low-HN cluster was rated as involving emotions more than the high-HN cluster. In sum, three out of five expectations about the characteristics of $\mathrm{HN}$ behaviors were met (see Table 5).

Contrasts between humans, animals, and robots

When rating the performance of behaviors by animals and robots, participants were given the option of nominating that a given behavior was not applicable to animals or robots by assigning a score of zero to it. When behavior-level ratings were calculated for these human versus nonhuman analyses, behaviors that received mean ratings of less than one were excluded from further analysis. Whereas $18 \%$ of the 60 behaviors received a mean rating less than one for animals,
$58 \%$ of the 60 behaviors received a mean rating of less than one for robots. Consistent with expectations, the correlation between $\mathrm{HU}$ and the behaviors that animals perform was $r=-.90, p<.01$, which means that the more a behavior was judged to involve HU, the less animals could perform it, relative to humans. Similarly, the correlation between HN and the behaviors that robots perform was $r=-.58, p<.01$, which means that the more a given behavior was judged to involve $\mathrm{HN}$, the less robots could perform it, relative to humans. There was no systematic relationship found between the $\mathrm{HN}$ ratings of behaviors and the animal behavior, $r=.28$, n.s. This means that animals were judged to be able to perform some HN behaviors more than humans and other HN behaviors less than humans. Similarly, there was no systematic relationship between robots and HU behavior, $r=-.13$, n.s. The behaviors that animals and robots were judged to perform either more or less than humans are presented in Table 6.

Table 5 Means and standard deviations of eight conceptual judgments across high-HU/low-HU and high-HN/low-HN clusters of behavior

\begin{tabular}{|c|c|c|c|c|c|c|}
\hline $\begin{array}{l}\text { Conceptual } \\
\text { judgment }\end{array}$ & $\begin{array}{l}\text { High-HU } \\
\text { cluster }\end{array}$ & $\begin{array}{l}\text { Low-HU } \\
\text { cluster }\end{array}$ & $\begin{array}{l}\text { Comparison of High } \\
\text { vs. Low HU }\end{array}$ & $\begin{array}{l}\text { High-HN } \\
\text { cluster }\end{array}$ & $\begin{array}{l}\text { Low-HN } \\
\text { cluster }\end{array}$ & $\begin{array}{l}\text { Comparison of High } \\
\text { vs. Low HN }\end{array}$ \\
\hline $\mathrm{HN}$ & $4.96(0.45)$ & $5.01(0.85)$ & $t(58)=-.18$, n.s. & $5.22(0.60)$ & $3.77(0.66)$ & $t(58)=6.61 * *$ \\
\hline HU & $\mathbf{5 . 7 1}(0.45)$ & $2.65(1.05)$ & $t(32.26)=14.87 * *$ & $3.10(1.61)$ & $3.47(0.75)$ & $t(23.61)=-1.10$, n.s. \\
\hline Positive & $5.81(0.44)$ & $4.82(1.80)$ & $t(55.36)=3.39^{*}$ & $5.44(1.24)$ & $2.39(1.58)$ & $t(58)=6.49 * *$ \\
\hline Emotional & $4.00(1.20)$ & $4.75(1.34)$ & $t(58)=-1.65$, n.s. & $4.51(1.38)$ & $5.31(0.84)$ & $t(16.92)=-2.37 * \mathrm{a}$ \\
\hline Universal & $5.53(0.65)$ & $\mathbf{6 . 0 2}(0.55)$ & $t(58)=-2.46^{*}$ & $\mathbf{6 . 0 2}(0.57)$ & $5.46(0.46)$ & $t(58)=2.79 *$ \\
\hline Prevalent & $5.23(0.82)$ & $5.17(1.36)$ & $t(58)=.14$, n.s. & $5.49(1.02)$ & $3.44(1.24)$ & $t(58)=5.36 * *$ \\
\hline Learned & $\mathbf{5 . 7 6}(0.46)$ & $4.21(0.95)$ & $t(26.99)=7.80 * *$ & $4.42(1.14)$ & $4.74(0.16)$ & $t(57.42)=-.83$, n.s. \\
\hline Maturity & $3.77(0.69)$ & $3.41(1.18)$ & $t(21.34)=1.32$, n.s. & $3.36(1.11)$ & $4.10(0.99)$ & $t(58)=-1.88$, n.s. \\
\hline Morality & $2.97(0.47)$ & $4.11(1.45)$ & $t(45.70)=-4.51 * * \mathrm{a}$ & $3.67(1.29)$ & $5.33(1.19)$ & $t(58)=-3.59^{*}$ \\
\hline Cognitive & $\mathbf{5 . 7 4}(0.81)$ & $4.19(1.21)$ & $t(58)=3.88^{* *}$ & $4.52(1.37)$ & $4.04(0.53)$ & $t(31.55)=1.85$, n.s. \\
\hline
\end{tabular}

$\mathrm{HU}$, human uniqueness; $\mathrm{HN}$, human nature. High-HU cluster is cluster $1(N=10$ behaviors). Low-HU cluster is the average of clusters $2-6(N=50$ behaviors). High-HN cluster is the average of clusters $1-5(N=51$ behaviors). Low-HN cluster is cluster $6(N=9$ behaviors $)$. Boldfaced means should be higher if indicator is valid

${ }^{\text {a }}$ Difference was opposite to expected direction

$* p<.05$

$* * p<.001$ 
Table 6 Behaviors that animals and robots perform less and more than humans

\begin{tabular}{|c|c|c|c|}
\hline \multicolumn{2}{|l|}{ Animals } & \multicolumn{2}{|l|}{ Robots } \\
\hline Less than humans & More than humans & Less than humans & More than humans \\
\hline Establishing affectionate relationships & Sex & Speaking/talking & Performing complicated tasks \\
\hline Paternal care for young & Sleeping & Locomotion & Performing calculations \\
\hline Verbal communication & Eating/drinking & Nonvocal communication & Performing repetitive tasks \\
\hline Nonvocal communication & Locomotion & Scanning the environment & Numerical reasoning \\
\hline Singing & Scanning the environment & Exploring & Serving others \\
\hline Making facial expressions & Exploring & Planning/organizing & Carrying objects \\
\hline Greeting gestures & Navigating obstacles in the environment & Teaching & Solving problems \\
\hline Judging others & Postures indicating triumph/surrender & Building shelter & Working \\
\hline Negotiating & Fighting & Navigating & Making things \\
\hline Planning & Impulsive aggression & Making music & Following rules \\
\hline Working & Hunting & Writing & \\
\hline Solving problems & Killing & Reading & \\
\hline Building shelter & & Growing food & \\
\hline Carrying objects & & Making art & \\
\hline Grasping objects & & Playing thinking games & \\
\hline Acting/pretending & & Making rules & \\
\hline Making music & & Grasping objects & \\
\hline \multicolumn{4}{|l|}{ Making rules Following rules } \\
\hline \multicolumn{4}{|l|}{ Complicated tasks } \\
\hline \multicolumn{4}{|l|}{ Helping family } \\
\hline \multicolumn{4}{|l|}{ Helping strangers } \\
\hline \multicolumn{4}{|l|}{ Forming social groups } \\
\hline \multicolumn{4}{|l|}{ Playing physical games } \\
\hline \multicolumn{4}{|l|}{ Serving others } \\
\hline \multicolumn{4}{|l|}{ Calculated aggression } \\
\hline \multicolumn{4}{|l|}{ Punishing } \\
\hline \multicolumn{4}{|l|}{ Bullying } \\
\hline \multicolumn{4}{|l|}{ Begging } \\
\hline \multicolumn{4}{|l|}{ Stealing } \\
\hline \multicolumn{4}{|l|}{ Performing repetitive tasks } \\
\hline Rape & & & \\
\hline
\end{tabular}

\section{Discussion}

This study examined people's humanness beliefs about behaviors, as well as judgments about the conceptual correlates of humanness beliefs. Using a range of prosocial, nonsocial, and antisocial behaviors, we found that people conceptualize behaviors along two distinct dimensions of humanness (HN, HU), that each sense of humanness is characterized by distinct conceptual profiles, and that $\mathrm{HU}$ and $\mathrm{HN}$ delineate humans from animals and machines, respectively. Taken together, these findings extend humanness research from the psychological traits and states that have been the focus of previous humanness research and consolidate our understanding of conceptions of humanness in folk psychology.
Conceptions of human nature

Consistent with prevailing conceptions of $\mathrm{HN}$ as a positive, innate, and universal sense of humanness, $\mathrm{HN}$ behaviors were judged as positive, prevalent within the population, and performed in all cultures. HN behaviors were also judged as not acquired through social learning, suggesting that the ability to perform $\mathrm{HN}$ behaviors is regarded as innate. These results were obtained both when $\mathrm{HN}$ behaviors were examined using correlational analyses and from comparisons of clusters of behaviors that were high and low on HN. However, contrary to expectations, $\mathrm{HN}$ behaviors were not judged as involving the experience of emotions, a conclusion borne out in both the correlational and cluster analyses. There are two reasons for this. First, behaviors that exemplified $\mathrm{HN}$ include basic 
nonsocial, self-preservative behaviors (e.g., sleeping, eating, drinking, locomotion) and complex prosocial, communal behaviors (e.g., sex, establishing and maintaining affectionate relationships, maternal care for young, forming social groups). Although both clusters of behavior exemplify $\mathrm{HN}$, the former are mechanical, automatic and unemotional, whereas the latter are more deliberative, attachment related, and emotional. This pattern of findings suggests that behavior type (e.g., nonsocial vs. prosocial) may moderate the $\mathrm{HN}-$ emotion relationship and that failure to consider this factor obscures it. Second, only $10 \%$ of the behaviors in the sample were judged as not part of $\mathrm{HN}$ (i.e., torturing, stealing, killing, begging, self-harm, and rape). Moreover, these six behaviors were judged as involving the experience of emotions $(M=5.63, S D=.63$; range: 5.18 6.48), which indicates that there was a potential restriction of range in emotionality ratings for non-HN behaviors. Hence, although our prediction about a general association between $\mathrm{HN}$ and emotionality was not supported by our data, our findings are not inconsistent with the idea that $\mathrm{HN}$ involves emotional responsiveness when behaviors are examined at a more granular level.

In addition to the pattern of conceptual judgments that were found for $\mathrm{HN}$, the results also support the idea that $\mathrm{HN}$ differentiates humans from inert nonhumans, such as robots and machines. Specifically, of those behaviors that robots were judged to perform, the higher the $\mathrm{HN}$ rating ascribed to the behavior, the less robots were judged able to perform it, relative to humans. Notably, there was no systematic relationship found between HU rating of behaviors and robot behavior. Unsurprisingly, many of the types of behaviors that robots were judged as able to perform either more (e.g., calculations, numerical reasoning) or less (e.g., navigating obstacles in the environment, scanning the environment for information) than humans mimic various information-processing capacities of humans.

\section{Conceptions of human uniqueness}

Consistent with prevailing conceptions of $\mathrm{HU}$ as a cognitively sophisticated sense of humanness that is acquired over time through the process of socialization into a specific culture, $\mathrm{HU}$ behaviors were judged as requiring maturity and thought, culturally specific, and acquired through social learning. Indeed, HU was most strongly associated with social learning, which suggests that this sense of humanness is understood as one that is acquired largely through socialization - a proposition consistent with traditional nurture arguments about human nature (see, e.g., Midgley, 1979/2000). These results for were obtained both when HU behaviors were examined using correlational analyses and from comparisons of clusters of behaviors that were judged as high and low on $\mathrm{HU}$, with the exception of maturity in the cluster-based analyses. However, the difference in the maturity required for the performance of high-HU behaviors was greater than that required for low-HU behaviors, albeit not significantly so. Contrary to expectations, HU behaviors were not judged as morally informative. The most likely explanation for this is that behaviors that exemplified HU were dominated by nonsocial behaviors that reflected uniquely human capacities for language and reason (i.e., reading, writing, speaking/talking, performing calculations, verbal and numerical reasoning). ${ }^{1}$ None of these behavioral exemplars of HU were seen as morally informative $(M=2.95, S D=.54$; range: $2.22-3.77)$.

The results also support the idea that HU differentiates humans from animals. Specifically, of those behaviors that animals were judged to perform, the higher the HU rating ascribed to the behavior, the less animals were judged able to perform it, relative to humans. Notably, there was no systematic relationship found between $\mathrm{HN}$ behaviors and animal behavior. Unsurprisingly, many of the types of behaviors that animals were judged to perform either more than (e.g., sex, sleeping), equal to (maternal care for young), or less than (e.g., helping family members, forming social groups) humans are behaviors grounded in our shared mammalian evolution.

\section{Implications of findings}

These findings will be useful to researchers of humanness, dehumanization, and anthropomorphism. First, the results of this study lend further support to Haslam's (2006) model of dehumanization and corroborate the proposition that lay conceptions of humanness, in Western countries, are characterized by two distinct dimensions: a species-typical sense (i.e., HN) and a species-unique sense (i.e., HU). Second, although information about the humanness of emotions, personality traits, and mental capacities is available (e.g., Demoulin et al., 2004; H. M. Gray et al., 2007; Haslam et al., 2005), there are no publically available indices of humanness beliefs about behavior. The availability of indices of behaviors organized along the dimensions of $\mathrm{HN}$ and HU significantly expands the range of validated materials available to researchers of humanness.

These findings will also be of use to researchers of social cognition more generally, particularly researchers of moral psychology. Although not hypothesized, our findings revealed a negative association between $\mathrm{HN}$ beliefs about behavior and

\footnotetext{
${ }^{1}$ Contrary to expectations, the behavior "verbal communication" $(M=$ $3.01, S D=2.01$ ) received significantly lower HU ratings than did "speaking/talking" ( $M=5.34, S D=1.88)$. These ratings indicate that speaking/talking was judged as uniquely human and that verbal communication was judged as nonuniquely human. Consistent with this, speaking/talking was ranked 7th on $\mathrm{HU}$ and 10th on $\mathrm{HN}$, and verbal communication was ranked 29th on HU and 6th on HN. This pattern of findings suggests that participants made a conceptual distinction between verbal communication and speaking/talking, such that the former was understood more as vocal communication, which is a behavior shared with other mammals, rather than uniquely human verbal communication, which is exemplified by speaking and talking.
} 
judgments about moral informativeness $(r=-.44, p<.01)$. This means that the more a behavior is part of human nature, the less informative it is about a person's moral nature. Moreover, judgments about the positivity or social desirability of behavior were negatively associated with morality judgments $(r=-.60, p<.01)$, which means that the more negative or socially undesirable a behavior is judged to be, the more it reveals about a person's moral character. Behaviors that exemplify this low-HN, negative profile include stealing, torturing, and killing. More generally, our results provide moral psychology researchers with an index of normative moral informativeness judgments for 60 prosocial, nonsocial, and antisocial behaviors (see supplemental file, Table 9), which complements extant indices of specific behaviors in the moral domain (see, e.g., Chadwick, Bromgard, Bromgard, \& Trafimow, 2006). The humanness and morality indices for general behaviors presented here provide useful information to researchers seeking to develop specific moral and nonmoral behavioral stimuli.

The humanness indices presented here will also be of use to attribution and social explanation researchers. For example, the distinct conceptual profiles of the two senses of humanness obtained in this study suggest that $\mathrm{HN}$ and $\mathrm{HU}$ behaviors may be associated with different types of behavior explanations. Malle's (1999) model of folk behavior explanation identifies two general classes of factors cited in the explanation of intentional behaviors-reasons (e.g., beliefs, desires) and causal history of reasons (e.g., personality traits) - which may be differentially associated with HN and HU behaviors. The results of this study suggest that many $\mathrm{HN}$ behaviors, such as sleeping, eating, and drinking, and perhaps maternal care of young, are viewed as innate. This suggests that explanations that make reference to causal history of reasons, or perhaps even unintentional cause-based explanations, may be more appropriate than reason explanations when making sense of $\mathrm{HN}$ behaviors. Conversely, the idea that $\mathrm{HU}$ behaviors are cognitively demanding suggests that cognitively sophisticated reason explanations are more appropriate for explaining HU behaviors than are causal history of reason explanations.

\section{Limitations}

Although an attempt was made to select a range of positive, neutral, and negative behaviors, the results indicate that the range of negative behaviors was limited. Moreover, whereas the positive behaviors sampled a range of mildly to highly positive behaviors, the negativity of the negatively valenced behaviors tended to be extreme (e.g., rape, self-harm), which may have biased the association between desirability and $\mathrm{HN}-$ evidenced by the strong zero-order correlation between $\mathrm{HN}$ and positivity $(r=.82)$. However, when the influence of the other theoretically relevant conceptual judgments was controlled for, the variance explained by the $\mathrm{HN}$-positivity relationship dropped from $67 \%$ to $31 \%$, suggesting that an unacceptable level of influence upon the HN-positivity relationship was not exerted by the inclusion of a small number of extremely negative behaviors.

An attempt was also made to sample a wide range of basic human behaviors that could be described at a similar level of abstraction. However, there were some highly abstract behaviors included in the sample (e.g., planning, verbal and numerical reasoning). Moreover, some of the behaviors were not specified very precisely (e.g., performing repetitive tasks, studying) or were compound behaviors involving a range of distinct behaviors (e.g., establishing and maintaining affectionate relationships, forming social groups). The abstraction of some of the behaviors included in the sample raises the possibility that some behaviors may have been subject to multiple "identities" (see, e.g., Vallacher \& Wegner, 1985, 1987) and subsequently identified at different "level" of construal to other behaviors. This is potentially problematic because, to the extent to which it occurred, it could have affected judgments about whether behavior reflects the two senses of humanness. Consider, for example, the behavior "manipulating an object." As explicated by Kozak et al. (2006), the lowest level of identification makes no reference to circumstances outside the action itself (e.g., "moving a finger"). However, this bodily movement can also be identified at a higher level that makes reference to the goals and purposes of the agent performing the action (e.g., "turning on a light"). In contrast to the first level of action identification, which makes no reference to mind or cognition and may, therefore, be judged as a basic HN behavior, the second level of identification may invoke cognition, particularly if a behavior is performed in the service of a conscious goal, potentially signifying $\mathrm{HU}$.

In sum, these results further corroborate the proposition that people distinguish between two distinct senses of humanness: a species-typical sense $(\mathrm{HN})$ and a species-unique sense (HU). Whereas $\mathrm{HN}$ is an innate sense of humanness that defines the boundary between humans and machines but is partly shared with animals, $\mathrm{HU}$ is a sense of humanness that defines the boundary between humans and animals but may be a locus of similarities with robots and machines. These senses of humanness both affirm and challenge traditional conceptions of humanness and contain within them the threat (or promise) of further eroding the boundaries of humanity.

\section{References}

Bandura, A. (2002). Selective moral disengagement in the exercise of moral agency. Journal of Moral Education, 31(2), 101-119. 
Bandura, A., Barbaranelli, C., Caprara, G. V., \& Pastorelli, C. (1996). Mechanisms of moral disengagement in the exercise of moral agency. Journal of Personality and Social Psychology, 71, 364-374.

Bandura, A., Underwood, B., \& Fromson, M. E. (1975). Disinhibition of aggression through diffusion of responsibility and dehumanization of victims. Journal of Research in Personality, 9, 253-269.

Bastian, B., Laham, S., Wilson, S., Haslam, N., \& Koval, P. (2011). Blaming, praising and protecting our humanity: The implications of everyday dehumanization for judgments of moral status. British Journal of Social Psychology, 50(3), 469-483.

Brooks, R. (2002). Flesh and machine: How robots will change us. New York: Pantheon.

Castano, E., \& Giner-Sorolla, R. (2006). Not quite human: Infrahumanization in response to collective responsibility for intergroup killing. Journal of Personality and Social Psychology, 90(5), 804-818.

Chadwick, R. A., Bromgard, G., Bromgard, I., \& Trafimow, D. (2006). An index of specific behaviors in the moral domain. Behavior Research Methods, 38(4), 692-697.

Demoulin, S., Leyens, J. P., Paladino, M. P., Rodriguez, R. T., Rodriguez, A. P., \& Dovidio, J. (2004). Dimensions of "uniquely" and "nonuniquely" human emotions. Cognition and Emotion, 18(1), 71-96.

Ekman, P. (1992). An argument for basic emotions. Cognition and Emotion, 18, 71-96.

Epstein, S. (1984). Controversial issues in emotion theory. Review of Personality and Social Psychology, 5, 64-88.

Fernández-Armesto, F. (2004). Humankind: A brief history. Oxford: Oxford University Press.

Goff, P. A., Eberhardt, J. L., Williams, M. J., \& Jackson, M. C. (2008). Not yet human: Implicit knowledge, historical dehumanization, and contemporary consequences. Journal of Personality and Social Psychology, 94(2), 292-306.

Gray, J. (2003). Straw dogs: Thoughts on humans and other animals. London: Granta.

Gray, H. M., Gray, K., \& Wegner, D. M. (2007). Dimensions of mind perception. Science, 315, 619.

Hampshire, S. (1991). Biology, machines, and humanity. In J. J. Sheehan \& M. Sosna (Eds.), The boundaries of humanity: Humans, animals, machines (pp. 253-256). Los Angeles, CA: University of California Press.

Haslam, N. (2006). Dehumanization: An integrative review. Personality and Social Psychology Review, 10(3), 252-264.

Haslam, N., Bain, P., Douge, L., Lee, M., \& Bastian, B. (2005). More human than you: Attributing humanness to self and others. Journal of Personality and Social Psychology, 89(6), 937-950.

Haslam, N., Bastian, B., \& Bissett, M. (2004). Essentialist beliefs about personality and their implications. Personality and Social Psychology Bulletin, 30, 1661-1673.
Kozak, M. N., Marsh, A. A., \& Wegner, D. M. (2006). What do I think you're doing? Action identification and mind attribution. Journal of Personality and Social Psychology, 90(4), 543-555.

Leyens, J. P., Paladino, M. P., Rodriguez, R. T., Vaes, J., Demoulin, S., \& Rodriguez, A. P. (2000). The emotional side of prejudice: The attribution of secondary emotions to ingroups and outgroups. Personality and Social Psychology Review, 4(2), 186-197.

Loughnan, S., \& Haslam, N. (2007). Animals and androids: Implicit associations between social categories and nonhumans. Psychological Science, 18, 116-121.

Loughnan, S., Haslam, N., \& Kashima, Y. (2009). Understanding the relationship between attribute-based and metaphor-based dehumanization. Group Processes \& Intergroup Relations, 12(6), 747-762.

MacLean, P. D. (1990). The triune brain in evolution. New York: Plenum Press.

Malle, B. F. (1999). How people explain behavior: A new theoretical framework. Personality and Social Psychology Review, 3(1), 23-48.

Midgley, M. (1979/2000). Beast and man. London: Routledge.

Plumwood, V. (2002). Environmental culture: The ecological crisis of reason. London: Routledge.

Rodriguez, R. T., Leyens, J. P., Rodriguez, A. P., Betancor Rodriguez, V., Quiles de Castillo, M. N., Demoulin, S., \& Cortés, B. (2005). The lay distinction between primary and secondary emotions: A spontaneous categorization? International Journal of Psychology, 40(2), 100-107.

Rothbart, M., \& Park, B. (1986). On the confirmability and disconfirmability of trait concepts. Journal of Personality and Social Psychology, 50(1), 131-142.

Somerville, M. (2006). The ethical imagination: Journeys of the human spirit. Melbourne: Melbourne University Press.

Turkle, S. (1991). Romantic reactions: Paradoxical responses to the computer presence. In J. J. Sheehan \& M. Sosna (Eds.), The boundaries of humanity: Humans, animals, machines (pp. 224252). Los Angeles, CA: University of California Press.

Vaes, J., Paladino, M. P., Castelli, L., Leyens, J. P., \& Giovanazzi, A. (2003). On the behavioral consequences of infra-humanization: The implicit role of uniquely human emotions in intergroup relations. Journal of Personality and Social Psychology, 85, 1016-1034.

Vallacher, R. R., \& Wegner, D. M. (1985). A theory of action identification. Hillsdale, NJ: Erlbaum.

Vallacher, R. R., \& Wegner, D. M. (1987). What do people think they're doing? Action identification and human behavior. Psychological Review, 94, 3-15.

Wilson, S., \& Haslam, N. (2009). Is the future more or less human? Differing views of humanness in the posthumanism debate. The Journal for the Theory of Social Behaviour, 39, 247-266. 